\title{
Digital Health Monitoring and Pervasive Healthcare Using Cloud- Connected Smart Wearable Devices
}

\author{
Tejas Upmanyu ${ }^{1}$, Shabbir Hussain ${ }^{1}$, Shivam Bharadwaj ${ }^{1}$ and Sandeep Saxena ${ }^{2}$ \\ ${ }^{1}$ GLBITM, Computer Science Department, Greater Noida, India \\ \{tejasupmanyu, pshabbir36, shivamb45\}@gmail.com \\ ${ }^{2}$ KEC, Computer Science Department, Ghaziabad, India \\ sandeep.s.in@ieee.org
}

\begin{abstract}
Mobile technology has made unprecedented progress in last 40 years, now combined with the unobtrusive power and efficiency of cloud computing. The next "...One more thing" in the technological arena from this intersection is "Digital Healthcare". Featuring state-of-the-art yet inexpensive medical data acquisition devices comprising of Network-connected sensors, either in the form of wearable smart devices or embedded in local environments. These sensors make very accurate medical data acquisition for prolonged time intervals forming a database in the cloud, rich of information and raw data for accurate analysis of the physical and medical health of individuals. Acquired continuously, collected in cloud, effectively processed and analyzed, such a technology could yield disruptive results in the field of medical diagnosis and research. In this work, we review the cosplay of mobile cloud computing with the pervasive IOT technology to redefine healthcare and present a promising vision for its future.
\end{abstract}

Keywords: wearable smart devices, mobile cloud computing, digital healthcare, remote health monitoring and fitness tracking

\section{Introduction}

Since the advent of mobile technology, 40 years ago, mobile devices have impacted human lifestyle on an unimaginable scale. From a humble but extraordinary beginning of voice calls to the humanistic touch of video calls, mobile technology not only revolutionized how we communicate but has affected our lives at the very fundamental level. Now being a natural extension of the individual, mobile technology has served a great relevance, carrying a much broader meaning and significance in today's world. Undoubtedly being one of the most admired achievements of the millennia, mobile devices have already transcended their initial shadow of being a just "mobile" communicating device, serving a far greater meaning to us today, as we look around, unbounded by space and time.

These devices are embedded with state-of-the-art sensors that are employed for personal health monitoring, fitness and awareness that work in a seamless manner with other smart devices, most popular being smartphones [1-3].

Besides alleviating fitness in a user-oriented manner, researchers have leaped ahead and have drawn serious implications of such technologies in health care and clinical research as remote health monitors for prolonged recording, analysis and management of patient's physiological signatures such as heart rate, sleep time, dietary requirements and so on [4-6]. Observing this pace of adoption of wearable technology [7], it would not be far when people would carry their personal medical data recorded over time by inexpensive wearable devices. which in turn will pre-process data, and aligning it in a specific structure then offloading it securely in encrypted form to cloud, using the rapidly emerging mobile cloud computing paradigm. Whenever you undergo a physical 
examination or seek medical attention, the doctor would have a rich and elaborate source of processed information regarding your health and related parameters on top of traditional lab-based tests.

Mobile Applications based on mobile ecosystems (iOS, Android, Windows Phone, etc.,) and remote servers provide a rich user experience fundamentally based on wireless networks. In the past decade, application stores of mobile devices have flooded with applications in a plethora of categories including entertainment, games, business, social networking, travel, news and so-on. There has been a recent emergence of a whole new class of applications - "Healthcare and Fitness" and recent trends show a great lot of interest in these applications [9]. Research Now also surveyed consumers who use medical apps and found that $96 \%$ said they believe the devices help them improve the quality of their lives [10]. There is practically no limit to what these applications can achieve and in the numerous ways they enhance a mobile user's Health and lifestyle.

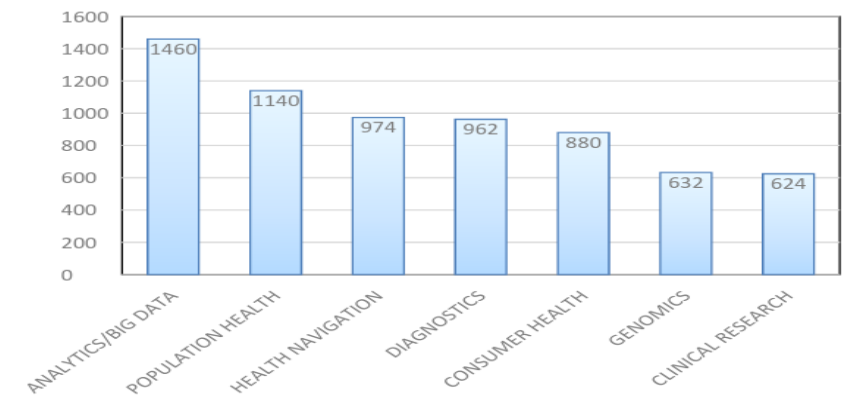

Figure 1. Investment in Different Fields of Digital Healthcare till 2015 in the US. (in USD Millions)

\section{Emerging Mobile Cloud Computing and its Tie-Up with IOT for Pervasive Healthcare}

Many Mobile devices providing medical sensors and that can detect various medical situations are not only available in the market but also being used by people today such as heart rate measurer, iris scanner, etc., are nowadays embedded into Smartphone's. Wireless technologies enable transmission of useful data via sensors (in mobile devices) to the caretakers. Also, machine to machine communications are nowadays common and enable communication with other devices, which is not limited to local but on cloud and the internet too. This intercommunication of devices over a network is referred to as Internet of Things. A possible medium for healthcare is IoT as discussed in [35, 36]. Data from the sensors will be stored in Cloud in processed forms of an appropriate structure such as tuples and attributes. This stored data will be analyzed by the respective doctor/ caretaker. Such an implementation has been proposed and demoed by [37].

\subsection{Scope of Mobile Cloud Computing}

As already stated in A Study of Mobile Cloud Computing: Architecture, Applications, and Challenges [8] and many other ongoing similar types of research researches that how Mobile cloud computing can provide a medium to compute with much power on remote places via portable small devices. With Mobile devices comes its perks and disadvantages.

Cloud computing platform provides pervasive healthcare computing feature [37] but still services like Amazon Web Services [42] and Microsoft Azure[43] things are becoming in our favor. The Concept of utilizing the MCC for pervasive healthcare is quite new but has great potential. 


\subsection{Related Approaches}

Although there are many similar types of researches going on, only a few of them use the cloud as the means of storing data, many of them would propose to use external storage devices such as SD cards and like but with growing network infrastructure around the globe and advances in that field Mobile Cloud seems to be an apt choice. It will provide practically unlimited storage space and a powerful and fast computing medium also it will allow the caretakers the mobility and easy access to the ( raw ) needed to them. There are some Cloud-based service providers which allows storing of sensors based data; the list includes Xively[38], Nimbits[39], ThingSpeak[40] and many more. Xively is a scalable infrastructure allowing users to deploy IoT-based services. It also features interfaces to make apps that can collect and process apps. Another related service is iDigi [41], it is a machine to machine PaaS (Platform-as-a-Service). It makes building costeffective, Secure and scalable solutions which make tying of enterprise level apps together with it.

\section{Background}

Almost all the proposed architectures and associated frameworks till date, for health tracking in a remote manner employ a general 3-tier organization, a WBAN (Wireless Body Area Network) which is comprised of wearables for the purpose of data gathering, a system for communicating data to the cloud and establishment of harmony among networked sensors and lastly a service layer [4] and [11-14]. For example, [15] presents a system that uses wearable devices to acquire a variety of different therapeutic situation like high blood pressure etc., These devices then pass the collected data to a gateway server through Bluetooth connectivity. An observation and measurement report is generated by the server, followed by storing it on a remote server, from where it can be easily accessed by medical research personnel or doctors via the internet. [16] Leverages a somewhat similar approach by storing medical data obtained in the cloud, from which cliniciens can obtain the saved data online using an application. WANDA [17] is an endto-end remote health tracking and analysis application, targeted towards patients with higher risks of heart malfunction.

Integrating IoT-based devices into such a system could produce advancements in intelligence, flexibility, scalability, accuracy and interoperability [13-18]. The advantages IoT devices carry with them is much significant as these devices will not only gather medical records more precisely and accurately but can also communicate with each other. This exchange of information among sensors can simplify administration tasks to great length. As illustrated in [19], in the case of an emergency such systems may directly report to the hospital staff, giving off early requests, which could save a lot of time and provide timely treatment to an individual.

\section{Architecture}

The Architecture depicted in Figure 2. Shows four major components of the system namely: (1) Data Gathering or Acquisition via wearable sensors on the patient. (2) Aggregators or concentrator devices such as a Smartphone. (3) Data storage in the cloud. (4) Clinician's application providing necessary interface to interact with the analyzed and visualized information already stored in the cloud. In the following text, we discuss the operations of these individual components. 


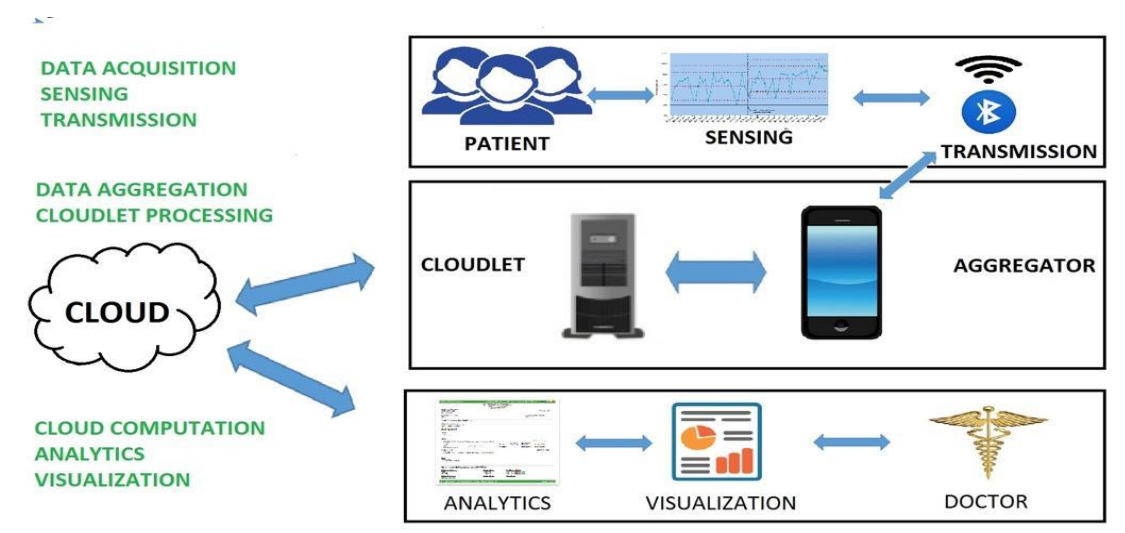

Figure 2. The Architecture of Remote Healthcare System, based on IoTCloud Collaboration

\subsection{Data Gathering/Acquisition}

Data Gathering/Acquisition is performed by a multitude of wearable devices comprised of sensors that measure physiological signatures, such as Sleep duration, Heartbeats, temperature, respiratory rate, muscle activity, and gait (posture). Wearable devices are a combination of a wide variety of sensors, which are capable of recording various physiological signatures, small pre-processing and communications hardware for transmitting the measured data. These devices connect to a Smartphone in proximity of the individual.

Connectivity of sensors to processors, instrumentation systems and control networks has been standardized by the IEEE 1451 family, including common network functions [22], wireless communication protocols [23], transducers to Radio Frequency Identification (RFID) Systems Communication Protocols [24]. On top of that, ISO IEEE 11073 personal health data standards family is also available for use in medical device communications, including specifications for communications through ECG monitoring [25], continuous glucose monitoring [26], and cardiovascular activity monitoring [27].

\subsection{Cloud Storage and Processing}

Medical data collected by the aggregator device is to be stored for long term usage; Cloud computing provides the best solution. Cloud-based storage offers countless benefits, major ones being: scalability, availability on demand, both for doctors and patients. Along with advanced techniques of visualization and analytics that work on the cloud, processing and hosting in the cloud can minimize costs to a great extent. In this subsection, we highlight such architectures and present issues that affect long-term storage on the cloud.

Hybrid Cloud/Cloudlet Architecture: Cloudlet is a Computing and storage platform with fixed resources that eliminates the need to offload resource intensive tasks to the enterprise cloud [32-33]. Cloudlet computing presents a potent answer to minimise latency in time-critical processes for health monitoring applications via body area networks [34]. The connection between aggregator device and nearest cloudlet is established using Wi-Fi technology. This direct link reduces latency in time critical tasks, dramatically for concentrated data. Long Term Evolution (LTE) technology made available in aggregator device may be utilized for concentrated data transfer from the aggregator device to the cloud storage, directly, bypassing the cloudlet, resulting in imposed latency due to slower mobile networks.

Smart Devices for context-aware aggregation of data: As in the preceding subsection, immigration of Wi-Fi technology along with recent LTE technology can result 
in leveraging of Smartphone's for use as the aggregator in IoT organization as an alternative. The concentration of data may be performed in any of the two ways: employing cloudlets via a Wi-Fi connection between aggregator device and a cloudlet, or by directly using cloud infrastructure through LTE enabled devices. Research indicates that using cloudlet as intermediate technology provides a throughput of up to ten times, compared to the LTE access to the cloud data. Along with that it also shows about onetenth of the access time. Cloud access via LTE also proves to be about twice as powerhungry as compared to cloudlets [39-45]. Whatever method be used, finally the recorded data must be stored in the cloud. Context- awareness can be put to use here, for effective division of medical data concentration among cloudlets and cloud. Use of Context allows clinicians to get aware of the present situation of the individual and also allows for accurate prognosis. For instance, while the patient is in a critical situation requiring monitoring of sensor data for immediate treatment, data aggregation should be the opted choice.

\subsection{Data Analytics and Visualization}

In comparison to laboratory grade instruments and the data they track that are used in subsequent diagnosis, wearable's can provide complete physical and metabolically measurements, acquired with state-of-the-art sensors that are capable of much accurate and precise sampling of medical data over prolonged periods of time. Such sets of rich medical data of individuals leave researchers with a treasure trove of information. Recent advancements in machine learning can provide relations among data acquired through sensors, and by analyzing this data over longer intervals spread over a large number of individuals can alleviate diagnoses.

It is not possible for doctors and researchers to work on the humongous datasets and their analyses received from sensors. To get the most out of the data collected by IoTbased sensors, data sets acquired and analyzed in the cloud must be formatted suitably before handing it over to clinicians at the other end, so that they can comprehend and study correlations among data received from different sensors, intuitively. Here enters the last stage of cloud processing, Visualization. It finds applications in routine life as well as scientific techniques, making it a significant research field [53]. Without a doubt, color plays a major part in shaping our visual sense. Visualization relies greatly on this theory. Studies show that variations in colours enhance comprehension and improve identification to spot differences in visualized data [54]. The best visualization may be obtained by determining the type of data. Data acquired from wearable sensors require a variety of visualization techniques. These datasets gathered are quite different from the data gathered by conventional laboratory methods as these are extracted over long intervals and are more accurate and precise samples of medical data.The data collected from these sensors is a goldmine of information for medical research and advancements in medicine, but before it proves to be fruitful to the clinicians and researchers, it must be formatted to impart comprehension and interactivity. The visualization technology seems to be a pivotal for the same. 
Table 1. List of Available and Future Sensors along with their Degree of Usability to Monitor and Detect Health Conditions in Concern with 3 Common Disease Classes: CARDIOVASCULAR DISEASES (CVD), CHRONIC OBSTRUCTIVE PULMONARY DISEASE (COPD), AND PARKINSON'S/HUNTINGTON'S DISEASES (PD). *** Indicates High Degree of usability, ** Indicates Some Degree of Usability, and * Indicates Uncertain Degree of Usability

\begin{tabular}{|l|l|l|l|l|}
\hline BIO MARKERS & $\begin{array}{l}\text { CARDIOVAS } \\
\text { CULAR } \\
\text { DISEASE }\end{array}$ & $\begin{array}{l}\text { CHRONIC } \\
\text { OBSTRUCTIVE } \\
\text { PULMONARY } \\
\text { DISEASE }\end{array}$ & $\begin{array}{l}\text { PARKINSON'S/HU } \\
\text { NTINGTON'S } \\
\text { DISEASES }\end{array}$ & DIABETES \\
\hline Gait (posture) & $* * *$ & $* * *$ & $* * *$ & $*$ \\
\hline ECG & $* * *$ & $* * *$ & $* *$ & $* *$ \\
\hline Respiratory rate & $* * *$ & $* * *$ & $* *$ & $* *$ \\
\hline Skin temperature & $* *$ & $* *$ & $* *$ & $*$ \\
\hline Surface EMG & $* *$ & $* *$ & $* *$ & $* *$ \\
\hline Sweating & $*$ & $*$ & $* *$ & $*$ \\
\hline Blood pressure & $* * *$ & $* *$ & $* *$ & $* * *$ \\
\hline Body movements & $* *$ & $*$ & $* *$ & $*$ \\
\hline Blood Glucose & $*$ & $*$ & $*$ & $*$ \\
\hline Heart Sound & $* *$ & $* *$ & $* *$ & \\
\hline Oxygenation & $* * *$ & $* * *$ & $* *$ & $*$ \\
\hline Title volume & $* * *$ & & $* *$ & $*$ \\
\hline & & & $* *$ & $*$ \\
\hline
\end{tabular}

\section{Issues and Challenges}

Mobile cloud computing based health care along with the integration of IoT technologies presents some challenges, which must be addressed effectively to tap into the full strength of this infrastructure.

\section{Large Data and Its maintenance and Structure:}

We have to deal with data which have high space and time complexity as a result of which data analyst works increase by a quite a fold. There is data visualization that could help in this kind of problem, but these techniques have been brought in light in recent years and requires extensive research and study before becoming feasible [44-45].

\section{Power Management and design of device in use:}

The design of sensors, if not comfortable to wear will create a physical limitation on the user, so there is a need that device is designed small, comfortable to wear, light, etc., so that they should not pose a problem in person movement. All these device requires power to run, As a result there is a great need that these devices must be power efficient. Battery inside those could be recharge and replace but in due process precaution must be taken such that data is not get corrupted or destroyed. Just making a device less power hungry will pose an entirely different problem. Problem like the data which is fetched is of very low quality and or get corrupted during sending. 


\section{Privacy of Data:}

Although we can remove the personal signature of data sent but still they pose a threat of disclosure attack that can infer information via pattern recognition approaches [46]. Techniques like Context-aware data concentration may provide a solution, but they too are vulnerable to disclosure attack by giving attacker to deduce one's health data by analyzing network traffic analysis from concentrator to mobile back haul.

\section{Secure Data Storage in the Cloud:}

Privacy is of great importance when data related to health is being stored in the cloud. According to the term defined by Health Insurance Portability and Accountability Act (HIPAA),the medical record must be protected from Divulging. When the dispatching of medical data in the cloud is done, appropriate privacy protection method must be taken to prevent access from unauthorized people, so the data privacy remains intact. There are many proposed framework for cloud storage to use with sensitive health records [51-53]. In spite of the data processing on the cloud is still an issue.

\section{Slow pace of Medical Instrument development:}

One of the challenges is there is a slow rate of progress in medical instrument. New Equipment and sensors require training of clinicians and regular approvals; this poses a constraint on the rate at which innovation is achieved. On the other hand, the rate at which the electronic circuitry is advancing is much faster and is governed by economic considerations to align closely with the so-called Moore's law. Machine Learning method requires further development so as to deal with such varied and regularly evolving sensors inputs.

\section{Heterogeneity:}

Maintaining mobility between different nodes connected in the cloud becomes difficult when they use different technologies like WiMAX, GPRS, WAP, WCDMA, etc., Such a situation creates a multitude of problems.

\section{Availability:}

The presence of various services that becomes a serious issue in Mobile Cloud Computing such as low-signal connectivity and network congestion. Huera-Canepa and Lee propose a solution to the problem of disconnection from clouds. They put a model which find a cloud-connected node around the range of a user. After this, application provider is changed. The main issue in the use of this technologies is security and concern of neighbour mobiles. Zhang et. al., [48-49] tries to solve this problem. In author propose Wi-Fi based multi-hop networking system called MoNet and a protocol for distributed content sharing in case of service unavailability. Every moving node acts as a distributor of messages containing info about device state like connectivity, storage, etc., regularly. On such regular notification, nodes can identify other nodes in the vicinity with lowest and highest resource hop [8].

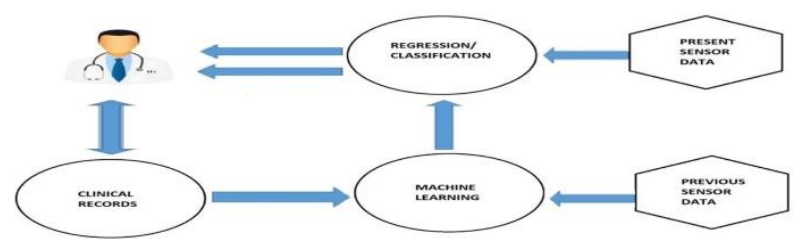

Figure 3. The Flow of Data after Analytics, on the Integration of Wearable Sensors 


\section{Conclusions}

Technologies with exponential growth in recent years, such as Internet of Things (IoT) and Mobile Cloud Computing (MCC) along with advancements in machine learning have made the dream of personalized and digitalized healthcare a reality. However, it's still away from its practical implementation on a wide scale all across the globe. Rocketing development of wearable smart devices with tons of functionality and aesthetics have paved the path for personalized health monitoring and awareness. It's high time to understand the merits and shortcomings of each of the involved technology to create an assembly that is simple, functional, spontaneous, reliable and pragmatic, with the harmonious relationship among all the technologies aimed at presenting the best possible experience to users at both the ends (patients and clinicians). We have made possible efforts to highlight each minor complication and concern whether it be security or privacy, in this paper. Along the way, we discussed the current trends and possible future directions for integration of IoT and cloud computing to offer unprecedented breakthroughs in medical diagnosis. Recently, IoT-enabled smart devices have surfaced, and a category which caught our attention is wearable's. These devices offer a ton of functionality along with pleasing aesthetics, making them an indispensable accessory in everyday life. Round the clock observation and acquisition of critical medical data in our local environments, piles up a goldmine of information, which when properly processed and presented neatly to the doctors, has a potential to change how we see diagnosis today. Besides the dramatic reduction in costs and improved efficiency of diagnosis, the data collected can offer a lot to medical research boosting its growth and in keeping up with new diseases and ailments that surface every other day. Along with that, such crucial data collected from billions of individuals who own a smart device can also increase the chances complete diagnosis for yet incurable diseases. Also, this technology can boost the quality of healthcare in developing nations.

\section{References}

[1] Apple Inc., “Apple watch,” accessed July 2016. [Online]. Available: https://www.apple.com/watch/

[2] FitBit Inc., "flex: Wireless activity + sleep wristband," accessed July 2016. [Online]. Available: https://www.fitbit.com/flex,

[3] Pebble Inc., "Pebble $2+$ Heart rate + sleep tracker," accessed July 2016 [Online]. Available: https://www.pebble.com/,

[4] A. Pantelopoulos and N. Bourbakis, "A survey on wearable sensor-based systems for health monitoring and prognosis," IEEE Trans. Sys., Man, and Cybernetics, Part C: Applic. and Reviews, vol. 40, no. 1, (2010) January, pp. 1-12.

[5] C. O. Milenkovi and E. Jovanov, "Wireless sensor networks for personal health monitoring: Issues and an implementation," Comput. Commun., vol. 29, no. 1314, (2006), pp. 2521 - 2533, wireless Sensor Networks and Wired/Wireless Internet Communications. [Online]. Available: http://www.sciencedirect.com/science/article/pii/S0140366406000508.

[6] R. Paradiso, G. Loriga and N. Taccini, "A wearable healthcare system based on knitted integrated sensors," IEEE Trans. Info. Tech. in Biomedicine, vol. 9, no. 3, (2005) September, pp. 337-344.

[7] Gartner Inc., "Gartner Says Worldwide Wearable Devices Sales to Grow 18.4 Percent in 2016" accessed July 2016 [Online]. Available: http://www.gartner.com/newsroom/id/3198018.

[8] T. Upmanyu, S, Bharadwaj and S, Saxena, "A Study of Mobile Cloud Computing: Architecture, Applications, and Challenges", IJCA Proceedings on Technical Symposium on Emerging Technologies in Computer Science TSETCS, vol. 2016, no. 1, (2016) June, pp. 10-17

[9] Acuant Inc., "Mobile App use for healthcare on the rise." accessed July 2016 [Online]. Available: http://www.acuantcorp.com/mobile-apps-for-healthcare-on-rise

[10] modernhealthcare.com, "Easy on those apps: Mobile medical apps gain support." accessed July 2016.http://www.modernhealthcare.com/article/20151128/MAGAZINE/311289981.

[11] R. Paradiso, G. Loriga and N. Taccini, "A wearable healthcare system based on knitted integrated sensors," IEEE Trans. Info. Tech. in Biomedicine, vol. 9, no. 3, (2005) September, pp. 337-344.

[12] C. T. Chou, R. Rana and W. Hu, "Energy efficient information collection in wireless sensor networks using adaptive compressive sensing," in IEEE 34th Conf.on Local Computer Networks, LCN 2009., (2009) October, pp. 443-450. 
[13] M. Bazzani, D. Conzon, A. Scalera, M. Spirito and C. Trainito, "Enabling the IoT paradigm in e-health solutions through the VIRTUS middleware," in IEEE 11th Int. Conf. on Trust, Security and Privacy in Computing and Com. (TrustCom), (2012) June, pp. 1954-1959.

[14] Benharref and M. Serhani, "Novel cloud and SOA-based framework for E-Health monitoring using wireless biosensors," IEEE Journal of Biomed. and Health Inf., vol. 18, no. 1, pp. 46-55, Jan 2014.

[15] S. Babu, M. Chandini, P. Lavanya, K. Ganapathy and V. Vaidehi, "Cloud-enabled remote health monitoring system," in Int. Conf. on Recent Trends in Inform. Tech. (ICRTIT), pp. 702-707, (2013) July.

[16] C. Rolim, F. Koch, C. Westphall, J. Werner, A. Fracalossi and G. Salvador, "A cloud computing solution for patient's data collection in health care institutions," in Second Int. Conf. on eHealth, Telemedicine, and Social Medicine, ETELEMED, no. 10, (2010) Febuary, pp. 95-99.

[17] M. Lan, L. Samy, N. Alshurafa, M. K. Suh, H. Ghasemzadeh, A. Macabasco-O'Connell and M. Sarrafzadeh, "Wanda: An end-to-end remote health monitoring and analytics system for heart failure patients," in Proc. of the Conf. on Wireless Health, ser. WH '12. New York, NY, USA: ACM, (2012), pp. 9:1-9:8.

[18] P. Ray, "Home health hub internet of things (H3IoT): An architectural framework for monitoring health of elderly people," in Int. Conf. on Science Eng. and Management Research (ICSEMR), (2014) November, pp. 1-3.

[19] N. Bui and M. Zorzi, "Health care applications: A solution based on the internet of things," in Proc. of the 4th Int. Symposium on Applied Sciences in Biomed. and Com. Tech., ser. ISABEL '11. New York, NY, USA: ACM, (2011), pp. 131:1-131:5.

[20] D. Son, J. Lee, S. Qiao, R. Ghaffari, J. Kim, J. E. Lee, C. Song, S. J. Kim, D. J. Lee, S. W. Jun, S. Yang, M. Park, J. Shin, K. Do, M. Lee, K. Kang, C. S. Hwang, N. Lu, T. Hyeon and D. H. Kim, "Multifunctional wearable devices for diagnosis and therapy of movement disorders," Nature Nanotechnology, (2014), pp. 1-8.

[21] S. Xu, Y. Zhang, L. Jia, K. E. Mathewson, K. I. Jang, J. Kim, H. Fu, X. Huang, P. Chava, R. Wang, S. Bhole, L. Wang, Y. J. Na, Y. Guan, M. Flavin, Z. Han, Y. Huang and J. A. Rogers, "Soft microfluidic assemblies of sensors, circuits, and radios for the skin," Science, vol. 344, (2014), pp. 70-74

[22] "IEEE Standard for a Smart Transducer Interface for Sensors and Actuators - Common Functions, Communication Protocols, and Transducer Electronic Data Sheet (TEDS) Formats," IEEE Std 1451.02007, (2007) September, pp. 1- 335.

[23] "IEEE Standard for a Smart Transducer Interface for Sensors and Actuators Wireless Communication Protocols and Transducer Electronic Data Sheet (TEDS) Formats," IEEE Std 1451.5-2007, (2007) October, pp. C1-236.

[24] "IEEE Standard for Smart Transducer Interface for Sensors and Actuators-Transducers to Radio Frequency Identification (RFID) Systems Communication Protocols and Transducer Electronic Data Sheet Formats," IEEE Std 1451.7-2010, (2010) June, pp. 1-99.

[25] "Health informatics-Personal health device communication Part 10406: Device specialization-Basic electrocardiograph (ECG) (1- to 3-lead ECG)," IEEE Std 11073-10406-2011, (2011) November, pp. 173.

[26] "Health informatics-Personal health device communication-Part 10425: Device SpecializationContinuous Glucose Monitor (CGM),” IEEE Std 11073-10425-2014, (2014) October, pp. 1-0.

[27] "Health Informatic-Personal health device communication Part 10441: Device specializationCardiovascular fitness and activity monitor," IEEE Std 11073-10441-2013 (Revision of IEEE Std 11073-10441-2008), (2013) March. pp. 1-108.

[28] D.-H. Kim, R. Ghaffari, N. Lu and J. A. Rogers, "Flexible and stretchable electronics for biointegrated devices," Annual Review of Biomedical Engineering, (2012), pp. 113-128.

[29] O. Olorode and M. Nourani, "Reducing leakage power in wearable medical devices using memory nap controller," in Circuits and Sys. Conf. (DCAS), IEEE Dallas, (2014) October, pp. 1-4.

[30] C. Park, P. Chou, Y. Bai, R. Matthews and A. Hibbs, "An ultra-wearable, wireless, low power ecg monitoring system," in Biomed.1 Circuits and Sys. Conf., BioCAS 2006. IEEE, (2006) November, pp. 241-244.

[31] T. Torfs, V. Leonov, C. Van Hoof and B. Gyselinckx, "Body-heat powered autonomous pulse oximeter," in 5th IEEE Conf. on Sensors, (2006) October, pp. 427-430.

[32] Y. Li and W. Wang, "The unheralded power of cloudlet computing in the vicinity of mobile devices," in IEEE Globecom Workshops (GC Wkshps), (2013) December, pp. 4994-4999

[33] M. Kwon, Z. Dou, W. Heinzelman, T. Soyata, H. Ba and J. Shi, "Use of network latency profiling and redundancy for cloud server selection," in Proceedings of the 7th IEEE International Conference on Cloud Computing (IEEE CLOUD 2014), Alaska, USA, (2014) June, pp. 826- 832.

[34] M. Quwaider and Y. Jararweh, "Cloudlet-based for big data collection in body area networks," in 8th International Conference for Internet Technology and Secured Transactions (ICITST), (2013) Decemebr, pp. $137-141$.

[35] V. M. Rohokale, N. R. Prasad and R. Prasad, "A cooperative Internet of Things (IoT) for rural healthcare monitoring and control", Wireless Communication, Vehicular Technology, Information 
Theory and Aerospace \& Electronic Systems Technology (Wireless VITAE), 2011 2nd International Conference, no, (2011) Febuary 28-March 3, pp. 1-6.

[36] A. Dohr, R. Modre-Opsrian, M. Drobics D. Hayn and G. Schreier, "The Internet of Things for Ambient Assisted Living", Information Technology: New Generations (ITNG), 2010 Seventh International Conference on, (2010) April 12-14, pp. 804-809.

[37] C. Doukas and I. Maglogiannis, "Bringing IoT and cloud computing towards pervasive healthcare," in Proc. 6th Int. Conf. Innov. Mobile Internet Services Ubiquitous Comput. (IMIS), (2012) July, pp. $922-$ 926.

[38] Xively by LogMeIn: IoT Platform for Connected Devices | Xively, https://www.xively.com/https://www.xively.com/

[39] Nimbits

Data Logging Cloud

Sever, http://bsautner.github.io/com.nimbits/http://bsautner.github.io/com.nimbits/

[40] Internet of Things - ThingSpeak service, http://www.thingspeak.comhttp://www.thingspeak.com/

[41] iDigi Device Cloud, http://www.idigi.comhttp://www.idigi.com/

[42] Amazon Web Services (AWS) - Cloud Computing Services, https://aws.amazon.com/https://aws.amazon.com/

[43] Microsoft Azure: Cloud Computing Platform and Services ,https://azure.microsoft.com/en-in/

[44] L. Wei, N. Kumar, V. Lolla, E. Keogh, S. Lonardi, C. Ratanamahatana and H. Van Herle, "A practical tool for visualizing and data mining medical time series," in Proc. 18th IEEE Symposium on ComputerBased Med. Sys., (2005) June, pp. 341-346

[45] Y. Mao, Y. Chen, G. Hackmann, M. Chen, C. Lu, M. Kollef and T. Bailey, "Medical data mining for early deterioration warning in general hospital wards," in IEEE 11th Int. Conf. on Data Mining Workshops (ICDMW), (2011) December, pp. 1042-1049

[46] Abbas and S. Khan, "A review on the state-of-the-art privacy preserving approaches in the e-health clouds," IEEE Journal of Biomedical and Health Informatics, vol. 18, no. 4, (2014) July, pp. 1431-1441

[47] M. Li, S. Yu, Y. Zheng, K. Ren and W. Lou, "Scalable and secure sharing of personal health records in cloud computing using attribute based encryption," IEEE Trans. Parallel Distrib. Syst., vol. 24, no. 1, (2013) January, pp. 131-143.

[48] X. Zhang, S. Jeong, A. Kunjithapatham and Simon Gibbs, -Towards an Elastic Application Model for Augmenting Computing Capabilities of Mobile Platforms,\| in The Third International ICST Conference on MOBILe Wireless MiddleWARE, Operating Systems, and Applications, Chicago, IL, USA, (2010).

[49] X. Zhang, J. Schiffman, S. Gibbs, A. Kunjithapatham and S. Jeong, -Securing Elastic Applications on Mobile Devices for Cloud Computing,\| in CCSW '09: Proceedings of the 2009 ACM Workshop on Cloud Computing Security. Chicago, IL, USA: ACM, (2009) November, pp. 127-134.

[50] Y. Jararweh, L. Tawalbeh, F. Ababneh and F. Dosari, "Resource efficient mobile computing using cloudlet infrastructure," in IEEE Ninth International Conference on Mobile Ad-hoc and Sensor Networks (MSN), (2013) December, pp. 373-377.

[51] M. Li, S. Yu, Y. Zheng, K. Ren and W. Lou, "Scalable and secure sharing of personal health records in cloud computing using attribute-based encryption," IEEE Transactions on Parallel and Distributed Systems, vol. 24, no. 1, (2013), pp. 131-143.

[52] S. Ruj, M. Stojmenovic and A. Nayak, "Privacy preserving access control with authentication for securing data in clouds," in Proceedings of the 2012 12th IEEE/ACM International Symposium on Cluster, Cloud and Grid Computing (Ccgrid 2012), ser. CCGRID '12. Washington, DC, USA: IEEE Computer Society, (2012), pp. 556-563.

[53] E. R. Tufte and P. Graves-Morris, "The visual display of quantitative information", Graphics press Cheshire, CT, vol. 2, (1983).

[54] C. Healey, "Choosing effective colours for data visualization," in Proceedings of the 7th conference on Visualization”, IEEE, (1996), pp. 263-270. 